\title{
EDUCACIÓN MEDIA Y SUPERIOR EN MÉXICO: ANÁLISIS TEÓRICO DE LA REALIDAD ACTUAL ${ }^{1}$
}

\author{
Oswaldo Lorenzo Quiles ${ }^{2}$ \\ José Édgar Zaragoza Loya ${ }^{3}$
}

\begin{abstract}
The Mexican educational system, one of the largest in the world, currently has different levels and types of training centers. Basic education consists of the community system with preschool, primary and secondary education. In high school, in addition to the general type, there is the technical secondary, telesecondary and secondary for workers, depending on the purpose to be achieved. For upper secondary education or, as it is called in other countries, Bachelor, there are also different types of studies. The student may opt for the general high school, the bivalent -mixture of technological and technical professional-, and the professional technical.

At this time, the Mexican educational system is trying to diversify the studies, offering more options and facilities for all social strata.

Basic education coverage hovers around $70-80 \%$ of the school age population. One of the biggest current challenges Mexican educational system faces is the implementation of compulsory education in mid-upper education which since 2012 aims to bring this level of education to all social classes, although its total coverage is not expected until 2021-2022 academic year.
\end{abstract}

Keywords: education system, Mexico, secondary and higher education, challenges of education

Resumen: El sistema educativo mexicano, es complejo por el alto número de alumnos a los que atiende, cuenta actualmente con distintos tipos de niveles y centros de formación. La educación básica está formada por el sistema comunitario en preescolar, educación primaria y educación secundaria. En secundaria, además del tipo general, se ubica la secundaria técnica, telesecundaria y secundaria para trabajadores, dependiendo de la finalidad que se persiga. En cuanto a la educación media superior o, como se denomina en el resto de países, Bachillerato, también existen distintas modalidades de estudios, pudiendo el alumno optar por el bachillerato general, el bivalente, mezcla del tecnológico y el profesional técnico.

En este momento, el sistema educativo mexicano trata de diversificar los estudios, ofreciendo mayores opciones y facilidades para todos los estratos sociales.

Acerca de la cobertura en educación básica, esta oscila alrededor del 70$80 \%$ de la población en edades escolares. Uno de los mayores retos actuales del sistema educativo mexicano es el de la implantación de la obligatoriedad en la educación media-superior, que desde el año 2012 pretende llevar este nivel educativo a todas las clases sociales, aunque su

Oswaldo Lorenzo Quiles; José Édgar Zaragoza Loya (2014). Educación Media y Superior en México: análisis teórico de la realidad actual. DEDiCA. REVISTA DE EDUCAÇÃO E HUMANIDADES, 6 (2014) março, 59-72 
cobertura total no se prevé hasta el año escolar 2021-2022.

Palabras Clave: sistema educativo; México; educación media y superior; retos de la educación

\section{Situación actual de la educación en México}

El actual sistema educativo mexicano es uno de los más grandes del mundo, siendo tercero en el continente americano y superado únicamente por Estados Unidos y Brasil. En 2009, el total de alumnos matriculados en los diferentes niveles educativos en Estados Unidos fue de 75.4 millones, en Brasil de 54 millones y en México 32.5 millones.

El sistema educativo nacional comprende tres grandes tipos de educación: la educación básica, la educación media superior y la educación superior.

Durante el ciclo escolar 2010/2011, se matricularon en total 38.384 .528 alumnos y casi el $90 \%$ de la matrícula total fue atendida por el sistema educativo escolarizado y un $10 \%$ por el extraescolar.

El sistema educativo escolarizado mexicano cuenta con 34.384.992 alumnos, 1.808.911 docentes y 253.661 escuelas de educación básica, media-superior y superior. En básica (preescolar, primaria y secundaria) están matriculados 25.666.451 alumnos, es decir, un $74.6 \%$ de la matrícula del sistema educativo escolarizado; en educación media-superior se registraron 4.187.528 estudiantes, que representan el $12.2 \%$ de la matrícula total; y en educación superior están inscritos 2.981.313 estudiantes, el $8.7 \%$ del total de estudiantes. Del total de docentes, el $65 \%$ pertenece a educación básica, el $15.4 \%$ a media superior y el $17.4 \%$ a superior.

La tabla 1 recoge el número total de alumnos matriculados, docentes y escuelas en función de los distintos niveles educativos.

\begin{tabular}{|c|c|c|c|}
\hline \multicolumn{3}{|c|}{ Porcentajes de alumnos matriculados en el periodo 2010-1011 } \\
\hline \multicolumn{3}{|c|}{ Escolarizado } & Extraescolar \\
\hline \multicolumn{3}{|c|}{$90 \%$} & $10 \%$ \\
\hline Ed. Básica & Ed. Media superior & Ed. Superior \\
\hline $74.6 \%$ & $12.2 \%$ & $8.7 \%$ & \\
\hline
\end{tabular}

Tabla 1. Porcentajes de la educación mexicana para el periodo 2010-2011 en función de los distintos tipos (elaboración propia basada en SEP, 2013)

Como se puede observar en la tabla anterior, el número de matriculados es claramente superior en la educación básica que en la educación media-superior y superior, lo que indica que no todos 
los egresados en educación básica continúan sus estudios superiores.

En cuanto a los porcentajes de matrícula de los diferentes niveles educativos del sistema de educación mexicano, quedaría distribuido de la siguiente manera (ver tabla 2):

\begin{tabular}{|c|c|c|c|}
\hline $\begin{array}{l}\text { TIPO } \\
\text { EDUCATI- } \\
\text { VO }\end{array}$ & $\begin{array}{c}\text { NIVEL } \\
\text { EDUCATIVO }\end{array}$ & $\begin{array}{c}\text { TIPOS DE } \\
\text { EDUCACIÓN } \\
\text { ESCOLARIZADA }\end{array}$ & $\begin{array}{c}\text { TIPOS DE } \\
\text { EDUCACIÓN } \\
\text { ESCOLARI- } \\
\text { ZADA }\end{array}$ \\
\hline \multirow{9}{*}{$\begin{array}{l}\text { Educación } \\
\text { básica }\end{array}$} & \multirow{2}{*}{ preescolar } & $\begin{array}{c}\text { Preescolar general } \\
\text { Preescolar comunitario }\end{array}$ & \\
\hline & & Preescolar indígena & \\
\hline & \multirow{3}{*}{ primaria } & Primaria general & \multirow{3}{*}{$\begin{array}{c}\text { Primaria para } \\
\text { adultos }\end{array}$} \\
\hline & & Cursos comunitarios & \\
\hline & & Primaria indígena & \\
\hline & \multirow{4}{*}{ secundaria } & Secundaria general & \multirow{4}{*}{$\begin{array}{c}\text { Secundaria } \\
\text { para adultos }\end{array}$} \\
\hline & & Para trabajadores & \\
\hline & & Secundaria Técnica & \\
\hline & & Telesecundaria & \\
\hline $\begin{array}{c}\text { Capacita- } \\
\text { ción para el } \\
\text { trabajo } \\
\end{array}$ & $\begin{array}{c}\text { Capacitación } \\
\text { para el } \\
\text { trabajo } \\
\end{array}$ & $\begin{array}{c}\text { Capacitación para el } \\
\text { trabajo }\end{array}$ & $\begin{array}{l}\text { Formación } \\
\text { para el } \\
\text { trabajo } \\
\end{array}$ \\
\hline \multirow{3}{*}{$\begin{array}{l}\text { Educación } \\
\text { Media } \\
\text { superior } \\
\end{array}$} & $\begin{array}{l}\text { profesional } \\
\text { técnico }\end{array}$ & Profesional técnico & \multirow{3}{*}{$\begin{array}{l}\text { Educación } \\
\text { abierta y a } \\
\text { distancia }\end{array}$} \\
\hline & \multirow{2}{*}{ bachillerato } & Bachillerato general & \\
\hline & & Bachillerato técnico & \\
\hline \multirow{6}{*}{$\begin{array}{l}\text { Educación } \\
\text { Superior }\end{array}$} & $\begin{array}{l}\text { Técnico } \\
\text { superior }\end{array}$ & Técnico superior & \multirow{6}{*}{$\begin{array}{l}\text { Educación } \\
\text { abierta y a } \\
\text { distancia }\end{array}$} \\
\hline & \multirow{3}{*}{ Licenciatura } & Educación normal & \\
\hline & & $\begin{array}{l}\text { Licenciatura } \\
\text { universitaria }\end{array}$ & \\
\hline & & $\begin{array}{l}\text { Licenciatura } \\
\text { tecnológica }\end{array}$ & \\
\hline & \multirow[b]{2}{*}{ Postgrado } & Especialidad & \\
\hline & & Maestría & \\
\hline
\end{tabular}

Tabla 2. Esquema general del sistema educativo nacional. Sistema educativo de los Estados Unidos Mexicanos (Zorrilla \& Barba, 2008)

\section{Educación media mexicana}

La educación media en México agrupa los diferentes niveles educativos que se abordan a continuación, en el marco del sistema educativo mexicano general.

Dentro de la educación media, la educación básica, o, como se denomina actualmente, la educación secundaria, es la comprendida entre los 12 y los 15 años de edad. Este nivel educativo es el que mayor número de población escolar tiene matriculada en México, con un $78 \%$ respecto a los otros tipos. 
En lo que respecta al tipo de servicio, el $85 \%$ de la población de educación básica asiste a centros estatales, el $7 \%$ de las escuelas pertenece a organismos federales y el $8 \%$ a escuelas privadas, por lo que un total del $92 \%$ de la población en la escuela básica iría a las escuelas públicas (SEP, 2012).

Desde el año académico 2002-2003, el 52\% de la población educativa ingresa en la educación secundaria general, el $20 \%$ a telesecundaria y el $28 \%$ a secundaria técnica. De estas tres opciones, la educación telesecundaria es la que muestra mayor incremento. Por otro lado, la secundaria técnica capacita a los alumnos en alguna actividad tecnológica industrial, comercial, agropecuaria, pesquera o forestal (SEP, 2013).

En la educación media, además de los servicios generales y comunitarios, existen secundarias técnicas, telesecundarias y secundarias para trabajadores. El plan de estudios de secundarias técnicas es muy parecido al de la secundaria general, pero da una mayor importancia a los conocimientos tecnológicos. Las telesecundarias, en cambio, surgieron en 1968 con el objetivo de luchar contra el atraso escolar y, sobre todo, en los ambientes rurales mediante un sistema de televisión educativa. En el sistema de la telesecundaria es un mismo profesor el que enseña todas las materias necesarias con la ayuda de materiales televisivos pedagógicos.

Por otra parte, la secundaria para trabajadores ofrece servicios a la población mayor de 15 años. Tras implantarse la obligatoriedad de la secundaria, será en este tipo de servicio donde el crecimiento es mayor. Así, de cada 100 alumnos que ingresan en educación secundaria, 47 lo harán en esta modalidad de secundaria para trabajadores (SEP, 2012).

El Gobierno Federal regula la asistencia de los alumnos a clase y la capacidad de gestión de las instituciones para que todos los egresados de educación primaria puedan alcanzar la educación secundaria.

Del total de población de alumnos que por edad deben y pueden estar escolarizados, son los alumnos indígenas los que menos asisten a clase.

\section{Educación media superior (Bachillerato) en México}

La educación media-superior, o bachillerato, complementa la enseñanza primaria superior, que capacitaría a los alumnos para vivir útilmente en la nación, y prepara a los alumnos para el ingreso 
a la universidad.

El sistema educativo mexicano sufre en 1995 una profunda reforma, tanto en su organización administrativa como en la curricular. Aparece el currículum basado en competencias y se intenta que los estudios cursados en el bachillerato respondan directamente a las necesidades del mercado laboral.

De los egresados en educación secundaria durante el ciclo escolar anterior a $2002-2003$, el $95.4 \%$ ingresa en el primer ciclo de educación media-superior. De los tres ciclos existentes en la educación media, el bachillerato general cubre el $60 \%$ de la población, cuya duración es de tres años, aunque puede haber casos excepcionales de dos o cuatro años.

El 29\% corresponde a alumnos de bachillerato tecnológico, que tiene las especialidades de tecnología industrial, de servicios, agropecuario, forestal y pesquero, y el restante $11 \%$ corresponde al bachillerato profesional técnico, el cual tiene como finalidad la formación para el trabajo y como opción seguir estudios superiores completando algunas asignaturas o elegir la opción de la inserción laboral.

El porcentaje de presencia de escuelas privadas puede pasar del $8 \%$ aproximadamente en educación básica al $22 \%$ en educación media superior, y continúa creciendo en los estudios superiores (SEP, 2013).

La educación media superior (EMS) consta de un solo nivel $y$, aunque los planes de estudio pueden variar entre dos y cinco años de duración, la edad habitual de los estudiantes de este nivel está entre 15 y 17 años (INEE, 2006; SEP, 2011).

La educación media superior está compuesta por el bachillerato general, bachillerato tecnológico y profesional técnico, mezclándose a veces alguno de ellos (SEP, 2011). A continuación se exponen los tres modelos comunes de bachillerato.

El bachillerato general o propedéutico ofrece una preparación general para que posteriormente los estudiantes puedan cursar educación superior. El alumno accede al estudio de las diferentes disciplinas humanísticas, científicas y tecnológicas con el objetivo de contar con experiencia que le pueda servir en sus estudios profesionales. El bachillerato general y propedéutico equivale al bachillerato general de otros países y cuenta con poco más del $60 \%$ del alumnado de la Educación Media Superior (SEP, 2013).

Existen también otros bachilleratos diferentes, como los 
bachilleratos universitarios y los generales, que no se encuentran vinculados a instituciones de educación superior, como los colegios de bachilleres o las escuelas preparatorias estatales.

El bachillerato bivalente, según Alcántara \& Zorrilla (2010), agrupa una parte de la Educación Media Superior Tecnológica junto con la Formación Profesional Técnica, y combina la formación técnica profesional para la incorporación al trabajo con la preparación para los estudios superiores. Es este un tipo de bachillerato por el cual los alumnos que completen su formación satisfactoriamente obtendrán dos títulos al terminar los estudios: uno que forma como profesional técnico capacitado para la incorporación laboral, reconocido por la Dirección General del Profesional, y otro que permite continuar con estudios superiores.

La educación profesional técnica atiende al $10 \%$ de la matrícula y ha sido tradicionalmente una formación sin pleno valor propedéutico. Desde 1997 hasta la actualidad, estos estudios permiten la equivalencia con el bachillerato superando seis cursos complementarios. Los títulos técnicos que ofrecen estas opciones tecnológicas son de calidad profesional y están registrados en la Dirección General de Profesiones de la Secretaría de Educación Pública (SEP), siempre y cuando se haya realizado la correspondiente tesis de grado y el servicio social.

Desde la creación de este nivel educativo de media superior, la matrícula ha crecido ininterrumpidamente y en los últimos años el número de estudiantes superó a los otros niveles que componen la educación básica.

A partir de octubre de 2010, el Pleno de la Cámara de Diputados del Gobierno Federal aprobó la obligatoriedad de la educación media-superior (Centro de estudios sociales y de opinión pública, 2011). Finalmente, en febrero de 2012 se firmó el decreto por el que se declaran reformados los artículos constitucionales que disponen que la obligatoriedad de la educación media superior será gradual y comenzará en el ciclo escolar 2012/2013 (Centro de estudios sociales y de opinión pública, 2012).

También en este caso el Gobierno de la República debe garantizar el acceso a este nivel educativo de toda la población escolar. Además, hay que tener presente que aún no se ha logrado universalizar los niveles escolares previos. Según los catos del INEE en 2010, todavía el 5\% de los egresados de primaria no continúa la educación secundaria, aun siendo ésta obligatoria, y 
sólo el $80 \%$ de los que terminan, lo hacen en tres años.

Según Velasco (2011), se calcula que en 2010, del total de jóvenes de 15 a 17 años que había concluido la educación secundaria, el $76 \%$ asistió a la educación media superior. Estos datos implican que 1.200.000 jóvenes enmarcados el rango de edad 15-17 y con educación básica terminada no asistía al bachillerato.

Por otra parte, el porcentaje de matrícula de alumnos de edades habituales que asisten a la educación media superior es hoy mayor al de los últimos diez años. Además, cabe destacar que en el periodo 2010-2011 este porcentaje es relativamente inferior al del año anterior. Sin embargo, estos valores siguen sin abarcar la cobertura total de escolaridad que el Gobierno mexicano persigue en este momento.

\section{Educación superior en México}

La educación superior es el tercer ciclo del Sistema Educativo Nacional mexicano. Comprende dos niveles, licenciatura y posgrado, y no tiene carácter obligatorio. El nivel licenciatura ofrece tres formas de servicio: licenciatura universitaria, licenciatura tecnológica y formación de maestros o educación normal.

Como indica Bollag (Bollag, 2009), el crecimiento vertiginoso producido en la educación superior por continuos cambios y movimientos de los jóvenes a otros lugares, gracias a la globalización de los estudios y a la posibilidad de recibir becas para cursar educación superior, hace que la matrícula de este tipo de educación no haya parado de crecer hasta la actualidad, aumentando a gran velocidad las inscripciones a la universidad y llegando en 2007 a un incremento de un 50\% de inscripciones respecto al año 2000.

La evolución de la matrícula y de la cobertura de educación superior en el ciclo 2006-2007 (sin considerar el posgrado) era ligeramente superior a 2.5 millones de alumnos. En el ciclo 20102011 la matrícula ya estaba por encima de los 3.000.000 y, de ese total, los alumnos que asisten a clase representan alrededor del 91\% (Ruiz, 2011; Tuirán, 2011).

Tuirán (Tuirán, 2011) expone que la matrícula de educación superior ha crecido no sólo en los programas impartidos bajo la modalidad escolarizada, sino también en la no presencial, en el nivel de Licenciatura. A su vez, la matrícula del posgrado ha 
aumentado igualmente de manera significativa.

La cobertura total de la educación superior (sin el posgrado) alcanzó en 2011 a más del 30\% de los jóvenes de 19 a 23 años.

En la figura 1 puede verse el aumento en la cobertura total y en la cobertura escolarizada a lo largo de los años hasta 2011.

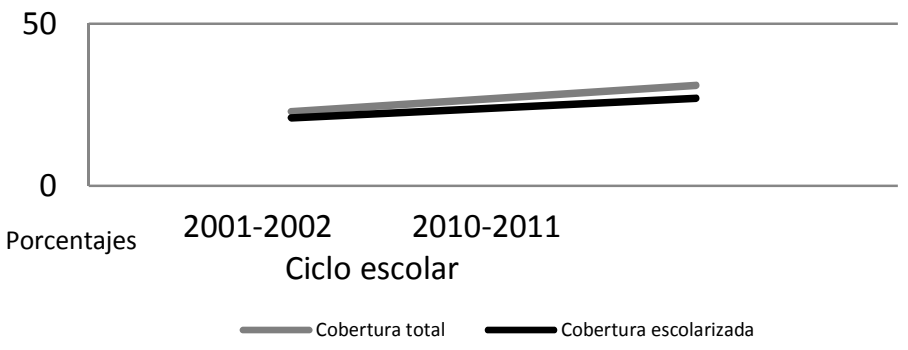

Figura 1. Evolución de la cobertura total y de la cobertura Escolarizada de la educación superior en México 2001-2010 (Subsecretaría de Educación Superior, 2010)

La ANUIES (Asociación Nacional de Universidades e Instituciones de Educación Superior en México) ha declarado su intención de elevar la meta de la cobertura de la educación superior al $48 \%$ en 2020 , lo que implicaría alcanzar una matrícula de alrededor de 4.700.000 estudiantes en ese año; es decir, aproximadamente 1.700 .000 jóvenes adicionales a la matrícula actual (Secretaría de Educación Superior -SEP, 2012). Para conseguir este objetivo, el ritmo de crecimiento debería ser por lo menos el mismo de los últimos dos ciclos escolares.

La meta de alcanzar la cobertura total tiene como objeto no sólo elevar el compromiso del régimen educativo público, como en años anteriores, sino el de aumentar a su vez el sistema privado. Así, habría que aumentar en 1.200.000 estudiantes el subsistema público y en 500.000 el privado.

El aumento de la matrícula de las instituciones públicas de educación superior implicaría un esfuerzo presupuestario sostenido de entre seis y siete mil millones de pesos adicionales cada año, y los programas de becas dirigidos a los jóvenes provenientes de familias de escasos recursos son y van a ser un instrumento eficaz para aumentar las posibilidades de acceso a -y 
permanencia en- la educación superior.

Tanto el Programa Nacional de Becas para la Educación Superior (creado en 2001) como otras iniciativas federales otorgaron en el ciclo 2006-2007 alrededor de 220.000 becas; éstas aumentaron a 387.000 en el ciclo 2009-2010, es decir, un incremento de alrededor de $75 \%$. En el ciclo 2010-2011 se otorgaron más de 425.000 becas a jóvenes de escasos recursos.

Los tres tipos de becas más importantes son las becas Pronabes, la beca Probapis y la beca de Fortalecimiento. La beca Pronabes (Programa Nacional de Becas) tuvo su origen en 20012002 y desde entonces se están concediendo con la finalidad de ayudar a los estudiantes de escasos recursos para que puedan completar sus estudios superiores. Las becas Probapis hacen referencia al programa beca de apoyo a la práctica intensiva y al servicio social para estudiantes de séptimo y octavo semestres de escuelas normales públicas. En cuanto a la beca de Fortalecimiento, ofrecida por la UNAM, está dirigida a los alumnos de licenciatura.

Todos estos tipos de beca tienen una serie de requisitos que cumplir y que guardan relación con la renta media familiar, una puntuación académica mínima, haber aprobado todas las asignaturas del curso anterior, ser mexicano, etc. Además, las becas son excluyentes, es decir, si se obtiene una no se puede obtener ninguna otra (SEP, 2012).

La matrícula actual de la educación superior, como ya se ha reflejado, no ha parado de aumentar en los últimos años y además se espera que ese crecimiento sea aún más rápido en los años siguientes. No obstante, el número de matrículas no es el mismo en todas las carreras.

En la figura 2 se observa cómo en los años 20062007/2009-2010 el número de personas matriculadas en algunas carreras ha descendido, debido probablemente al aumento de oferta de estudios y la diversificación de éstos que se dio durante el periodo analizado (SEP, 2013). 


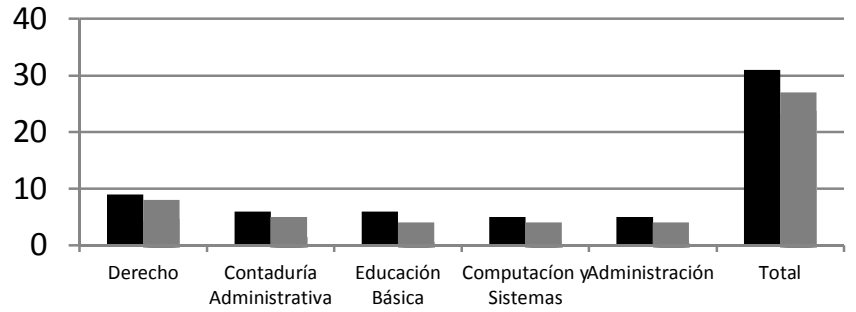

Figura 2. Porcentaje de la matrícula total de educación superior representada por las cinco carreras con mayor número de estudiantes, ciclos 2006-2007 a 2009-2010 (Secretaría de Educación Superior, 2011)

El aumento de las matrículas en educación superior y las becas otorgadas para favorecer el seguimiento de estos estudios por personas con escasos recursos económicos tiene, sin duda, efectos positivos en el país, lo que ha hecho que el nivel de inversión federal por estudiante en este nivel crezca notablemente en los últimos dos años.

La expansión cuantitativa de la educación superior durante las décadas de los sesenta y setenta no se acompañó de reformas de fondo adecuadas a este nivel educativo y su modelo académico (Rodríguez, 2002). Por el contrario, el crecimiento de este tipo de educación se llevó a cabo bajo una estructura tradicional que no produjo la calidad deseable. Así, entre 1980 y 1990 la población universitaria pasa de 838.000 a 2.241 .000 alumnos. Si la población total del país en el año 1980 es de 87.000.000 de habitantes y de ésta el $10 \%$ corresponde a la franja de edad de 20-24 años, esto significa que sigue estudios superiores uno de cada cuatro jóvenes en el rango 20-24 años, es decir, el 25\% de esta franja de edad (Instituto de Estudios Educativos y Sindicales de América -IESSA, 2012).

El número de instituciones de educación superior que presenta el país entre 1995-2000 es de 1.051, siendo mayor el número de estas de carácter público que privado. En cuanto al tipo de subsistema al que se puede optar en educación superior, el subsistema universitario general es el que predomina claramente por encima del resto. 
La distribución de matrícula entre las diferentes instituciones y tipo de educación, para los ciclos 1999-2000 y 2000-2001, cuenta con el mismo resultado, teniendo la educación pública mayor número de alumnos que la universidad privada.

De acuerdo con el Programa de Desarrollo Educativo 19952000 , el sistema de educación superior mexicano se articulaba del siguiente modo (ver tabla 3 ):

\begin{tabular}{|l|l|l|l|l|l|l|l|}
\hline \multicolumn{2}{|c|}{ Instituciones } & $\begin{array}{c}\text { Total de } \\
\text { instituciones }\end{array}$ & \multicolumn{4}{|c|}{ Subsistemas } & Otros \\
\hline $\begin{array}{l}\text { Públi } \\
\text {-ca }\end{array}$ & $\begin{array}{l}\text { Pri- } \\
\text { vada }\end{array}$ & & Universitario & $\begin{array}{l}\text { Tecnoló- } \\
\text { gico }\end{array}$ & $\begin{array}{l}\text { Universi- } \\
\text { tario- } \\
\text { tecnológi } \\
\text { co }\end{array}$ & $\begin{array}{l}\text { Nor- } \\
\text { mal }\end{array}$ & \\
\hline 834 & 217 & 1.051 & $68 \%$ & $17.2 \%$ & $0.3 \%$ & $9 \%$ & $5.5 \%$ \\
\hline
\end{tabular}

Tabla 3. Composición del sistema de educación mexicano en la educación superior (1995-2000) (SEP, 2013)

Los estudios de Técnico Superior se imparten principalmente en Universidades Tecnológicas, impulsadas por los servicios federales desde 1991, que se ocupan de la administración de este nivel en un $64.5 \%$.

Dentro de las Licenciaturas se ubica la educación normal, que cubre el $8.2 \%$ de la matrícula y en ésta se imparten en las especialidades de preescolar, primaria, secundaria, educación especial, atención de niños con discapacidad o capacidades especiales y educación física (SEP, 2012).

\section{Conclusiones}

La revisión y el análisis teórico aquí hechos sobre el sistema educativo mexicano de educación media y superior permiten observar una densidad y complejidad importantes en el contexto político y social en el que éste tiene lugar. Parece necesario que la Secretaría de Educación Pública de la república de México acometa sin dilación medidas de dimensionamiento de un sistema educativo demasiado centralista en su gestión y poco diversificado, lo que conllevaría un mejor y mayor acceso a la educación media-superior y, especialmente, a la superior, a todos los estratos sociales.

La necesidad de una mayor proyección social de la educación mexicana viene dada por la evidencia de que un país en constante crecimiento, con una población de más de cien millones de habitantes, requiere de sistemas de gestión educativa flexibles 
que faciliten e incentiven la continuidad y permanencia de los estudios en el tramo de educación media-superior, pronto un tramo educativo obligatorio, y ofrezcan estímulos a la continuidad de estos estudios en la educación superior, nivel al que sólo una pequeña parte de la población accede y que garantiza en los diferentes países del mundo una ciudadanía crítica, competente y comprometida con el desarrollo de toda la sociedad.

En el caso de la educación media-superior, el Gobierno de la República debe garantizar el acceso a este nivel educativo de toda la población escolar, y hay que tener presente que aún no se ha logrado universalizar los niveles escolares previos. Según el INEE (2010), todavía el $5 \%$ de los egresados de primaria no continúa la educación secundaria, aun siendo ésta obligatoria, y sólo el $80 \%$ de los que terminan lo hacen en tres años. Así, uno de los mayores retos actuales del sistema educativo mexicano es el de la implantación de la obligatoriedad en la educación media-superior, que desde el año 2012 pretende llevar este nivel educativo a todas las clases sociales, aunque su cobertura total no se prevé hasta el año escolar 2021-2022.

Lo anterior es un paso previo necesario para el aumento de la matrícula de las instituciones públicas de educación superior, que implicaría un esfuerzo presupuestario sostenido de millones de pesos cada año por la SEP, sosteniendo además programas de becas dirigidos a los jóvenes provenientes de familias de escasos recursos, un instrumento básico y eficaz para aumentar las posibilidades de acceso a -y permanencia en- la educación superior.

\section{Referencias/ Bibilografía}

Alcántara, A.; Zorrilla, J. F. (2010). Globalización y Educación media superior en México. En busca de la perspectiva curricular. Perfiles educativos, 32, 127 (2010) 38-57. Consultado en 7 de marzo de 2014. Disponible en: http://www.scielo.org.mx/pdf/peredu/v32n127a3.pdf

Bollag, B. (2009). Grandes cambios sacuden la educación superior. El hombre y la naturaleza: vivir en armonía. El correo de la UNESCO, 6, 1 (2009) 17. Consultado en 7 de marzo de 2014. Disponible en: http://unesdoc.unesco.org/images/0018/001867/186704s.pdf

Centro de estudios sociales y de opinión pública (Cámara de diputados) (2011). Informe sobre la educación superior en México. Consultado en 7 de marzo de 2014. Disponible en: http://www.diputados.gob.mx/cesop/doctos/DDL040\%20Informe\%20sobre $\% 201 a \% 20$ educacion\%20superior

Centro de estudios sociales y de opinión pública (Cámara de 
diputados, LIV Legislatura) (2012). Informe sobre la educación superior en México. Consultado en 7 de marzo de 2014. Disponible en: http://www3.diputados.gob.mx/camara/001_diputados/006_centros_de_est udio/05_centro_de_estudios_para_el_adelanto_de_las_mujeres_y_la_equ idad_de_genero/001b_boletin_mensual

Instituto de Estudios Educativos y Sindicales de América -IESSA (2012). Evolución del gasto en educación en México. Consultado en 7 de marzo de 2014. Disponible en:

http://www.ieesa.org.mx/Datos/Evolucion_del_gasto_en_educacion_en_M exico.pdf

INEE -Instituto Nacional para la Evaluación de la Educación(2010). El Derecho a la Educación en México. Informe 2009. México DF: INEE.

Rodríguez, R. (2002). Continuidad y cambio de las políticas de Educación Superior. Revista mexicana de investigación educativa, 7, 14 (2002) 133-154. Consultado en 7 de marzo de 2014. Disponible en: http://redalyc.uaemex.mx/pdf/140/14001407.pdf.

Ruiz, E. (2011). La educación superior tecnológica en México. Historia, situación actual y perspectivas. Revista lberoamericana de Educación Superior (RIES), 2, 3 (2011) 35-52.

SEP (2012). Sistema educativo de los Estados Unidos Mexicanos, principales cifras. Consultado en 7 de marzo de 2014. Disponible en: http://www.sep.gob.mx/work/models/sep1/

Resource/1899/2/images/principales_cifras_2011_2012.pdf

SEP (2013). Sistema educativo de Ios Estados Unidos Mexicanos, principales cifras. Consultado en 7 de marzo de 2014. Disponible en: http://www.snie.sep.gob.mx/estadisticas_educativas.html

Resource/1899/2/images/principales_cifras_2011_2012.pdf

Tuirán, R (2011). La educación superior en México: avances, rezagos y retos. Consultado en 7 de marzo de 2014. Disponible en: ftp://ece.buap.mx/pub/TRANSPARENCIA/PlanDesarrollo FCE_1115/Educacion/EducSupMex_AvancRezagosRetos-SEP.pdf

Velasco, J (2011). La Educación Media Superior en México. Instituto Nacional para la Evaluación de la Educación (INEE). Consultado en 7 de marzo de 2014. Disponible en:

http://www.inee.edu.mx/images/panorama2011/version13092012.pdf

Zorrilla, M.; Barba, B. (2008). Reforma educativa en México. descentralización y nuevos actores. Sinéctica - Revista electrónica de Educación. Consultado en 7 de marzo de 2014. Disponible en: http://www.sinectica.iteso. $\mathrm{mx} /$ ? seccion=articulo\&lang=es\&id=441_reforma _educativa_en_mexico_descentralizacion_y_nuevos_actores

\footnotetext{
${ }^{1}$ Secondary and Higher Education in Mexico: theoretical analysis of the current situation

${ }^{2}$ Doctor.

Universidad de Granada (España).
} 
E-mail: oswaldo@ugr.es

${ }^{3}$ Doctor.

Universidad Autónoma de Tamaulipas (México).

E-mail: zaragoza@uat.edu.mx 